evidence on effects of early and delayed cord clamping in preterm infants.

\section{TISSUE OXYGENATION IN THE LIGHT OF NON-INVASIVE AND CONTINUOUS NEAR-INFRARED SPECTROSCOPY AND IMAGING (NIRS, NIRI)}

doi:10.1136/archdischild-2012-302724.0022

M Wolf. Division of Neonatology, University Hospital Zurich, Zurich, Switzerland

Near-infrared spectrophotometry (NIRS) and imaging (NIRI) are quickly growing optical methods to non-invasively and in vivo study oxygenation of human tissue. NIRS and NIRI are appreciated by patients, relatives e.g. parents, medical personnel and researchers because the methods are harmless, painless, quantitative, bedside applicable, enable continuous measurements (monitoring) and are thus well suited even for fragile and vulnerable intensive care patients.

The presentation will briefly explain the main principles of NIRS and NIRI, including the parameters that can be measured. The relevance of NIRS/NIRI measurements in research and clinics and also potential pitfalls will be discussed.

Then the main applications of NIRS and NIRI in neonatal medicine will be reviewed, e.g. measurements of the brain to avoid hyper- or hypo-oxygenation to safeguard the brain or to study brain activity and function and peripheral measurements, e.g. liver, gut, muscle. The state of these applications and their validity will be addressed.

An increasing number of commercial NIRS instruments is available and an overview will be given.

In an outlook future technical developments, which will enable to non-invasively measure other clinically important parameters such as blood flow, cytochrome oxidase redox state, water and lipids will be presented. Finally, the state of the art in tomographic NIRI with continuously increasing spatial resolution will be presented.

\section{UTILITY OF MICROCIRCULATION ANALYSIS IN A PAEDIATRIC ANIMAL MODEL OF HYPOVOLEMIC SHOCK}

doi:10.1136/archdischild-2012-302724.0023

1.2R Gonzalez, ' $\mathrm{J}$ Urbano, ' $\mathrm{J}$ Lopez, 1,2MJ Solana, 'A García, 'M Botran, '1 López-Herce. 'Pediatric Intensive Care, Gregorio Marañón General University Hospital and Research Institute; ${ }^{2 H e a l t h ~ R e s e a r c h ~ F o u n d, ~ H e a l t h ~ I n s t i t u t e ~ " C a r l o s ~ I I I ", ~ M a d r i d, ~ S p a i n ~}$

Background and aims Evaluation of tisular perfusion is very important in critically ill patients. Several techniques are used to assess tisular blood flow. Most of them are invasive and non accurate. Sidestream dark field imaging is proposed to be a useful noninvasive method to evaluate microcirculation.

Methods Prospective, observational study in 17 two month-old piglets $(8.6 \pm 1.1 \mathrm{~kg})$. Following mechanical ventilation, hypovolemic shock was induced by controlled $30 \mathrm{ml} / \mathrm{kg}$ bleed. 5 video sequences were recorded in each pig at basal time, during shock, and after fluid resuscitation using Microscan Microvision ${ }^{\circledR}$ device. Recorded video sequences were analysed later. Microcirculation was assessed determining perfused vessel density (PVD), microvascular flow index (MFI) and heterogeneity index (HI). Automated vascular analysis (AVA®) software was used to analyze the sequences.

Results Before bleed median values for PVD $(13.6 / \mathrm{mm} 3 \pm 2.4)$ and MFI (2.75 \pm 0.22$)$ where higher and HI $(0.21 \pm 0.14)$ was lower than during shock $(12.4 / \mathrm{mm} 3 \pm 1.4,1.97 \pm 0.43$ and $0.58 \pm 0.36$ respectively) $(\mathrm{p}<0.05)$. After fluid resuscitation PVD and MFI median values increased $(13.69 / \mathrm{mm} 3 \pm 1.56$ and $2.63 \pm 0.26$ respectively) and $\mathrm{HI}$ decreased $(0.32 \pm 0.22)(p<0.05)$.

Conclusions Lower vessel density and slower microvascular blood flow and higher flow heterogeneity occurred during shock. Computerized microcirculation analysis using sidestream dark field was able to distinguish between basal condition and hypovolemic shock and between hypovolemic shock and after fluid resuscitation.

\section{CORRELATION OF MICROCIRCULATION ANALYSIS WITH HEMODYNAMIC AND BIOCHEMICAL PARAMETERS OF TISULAR PERFUSION}

doi:10.1136/archdischild-2012-302724.0024

1,2R Gonzalez, 'J Urbano, 1'2MJ Solana, 'J López, 'M Botrán, 'A García, 'J Del Castillo, 'J López-Herce. 'Pediatric Intensive Care, Gregorio Marañón General University Hospital and Research Institute; 'Health Research Found, Health Institute "Carlos III", Madrid, Spain

Background and aims Microcirculation assessment focus on 3 components: Vessel density, perfusion, and heterogeneity of microcirculation, measured as perfused vessel density (PVD), microcirculation flow index (MFI), proportion of perfused vessels and heterogeneity index (HI). Correlation of these parameters with hemodynamic and perfusion parameters is not well established.

Methods Prospective, observational study in 17 two-month-old sedated, relaxed and mechanically ventilated piglets $(8.6 \pm 1.1 \mathrm{~kg})$. Video sequences were recorded using Microscan Microvision ${ }^{\circledR}$ device at three different times: before, after induced hypovolemic shock and after fluid resuscitation. 51 sets of measurements where obtained by the analysis of video sequences using automated vascular analysis software. Microcirculation was assessed determining PVD, MFI and HI

Results PVD showed correlation with MFI (r: 0,589) and central venous oxygen saturation $(\mathrm{SvO} 2)(\mathrm{r}: 0,383)$ and $\mathrm{HI}(\mathrm{r}:-0,600)$ (all $\mathrm{p}<0.01)$. MFI showed correlation with PVD ( $\mathrm{r}: 0,589)$, systolic ( $\mathrm{r}: 0,540)$, diastolic ( $\mathrm{r}: 0,443)$, and median ( $\mathrm{r}: 0,517)$ blood pressure, cardiac index (CI) (r:0,578), SvO2 (r:0,462), internal carotid artery flow (ICAF) (r:0,623) HI (r:-0,864), lactate blood levels (r:-0,476) (all $\mathrm{p}<0.01)$, and intramural gastric $\mathrm{pH}(\mathrm{r}: 0,352)(\mathrm{p}: 0,028)$

HI showed correlation with PVD ( $\mathrm{r}:-0,600)$, MFI (r:-0,864), systolic ( $\mathrm{r}:-0,359)$ and median ( $\mathrm{r}:-0,350)$ blood pressure, CI ( $\mathrm{r}:-0,389)$, arterial $\mathrm{pH}(\mathrm{r}:-0,458), \mathrm{SvO} 2(\mathrm{r}:-0,492)$, ICAF $(\mathrm{r}:-0,458)($ all $\mathrm{p}<0.01)$, systemic vascular resistance index (r:0,316) (p:0,027) and diastolic blood pressure ( $\mathrm{r}:-0,291)$ ( $\mathrm{p}: 0,038)$.

Conclusions Microcirculation parameters (PVD, MFI and IH) were consistent and related to global hemodynamic and tissue perfusion parameters.

\section{CAN LOW PERFUSION INDEX PREDICT THE TREATMENT NEED IN PREMATURE INFANTS WITH PATENT DUCTUS ARTERIOSUS?}

doi:10.1136/archdischild-2012-302724.0025

'D Terek, ${ }^{10}$ Altun Koroglu, ${ }^{10}$ Uygur, ${ }^{2} Z$ Ulger, 'M Yalaz, 'N Kultursay. 'Neonatology; 2Pediatric Cardiology, Ege University Faculty of Medicine, Izmir, Turkey

Background and aims Perfusion index (PI) shows real time changes in peripheric blood flow. Among critically ill infants, it predicts poor perfusion and the severity of the disease. Early diagnosis and treatment of PDA is important to prevent complications due hemodynamically significant patent ductus arteriosus (PDA). In this study, we aimed to compare the PI values of premature infants with and without hemodynamically significant PDA.

Methods Forty one premature infants were evaluated with echocardiography at the postnatal days 0 and 3 . Patients were grouped as: Group $1(\mathrm{n}=19)$ : no - PDA; Group $2(\mathrm{n}=10)$ hemodynamically nonsignificant PDA; Group $3(\mathrm{n}=12)$ hemodynamically significant PDA. PI was measured during a quiet state at the postnatal days 0 , 1, 2 and 3 by Masimo pulse oximeter. Clinical characteristics of the infants were recorded prospectively.

Results All the study groups were similar with regard to birth weight (1473 \pm 51 grams) and gestational age (30 \pm 2.9 weeks). Group 
3 had significantly lower day 0 PI values compared to Group 1 and 2 $(p=0.008)$. PI values of Group 3 increased after ibuprofen treatment and became similar to Group 1 and 2 after PDA closure on the postnatal days 2 and 3 .

Conclusion PI values of infants with hemodynamically significant PDA were lower at postnatal day 0 and with ibuprofen treatment; PI values increased to levels of infants without significant PDA. Our data show that PI is an early and noninvasive parameter predicting poor perfusion and may be helpful in decision making for PDA closure

\section{IMPACT OF SEVERITY OF PERINATAL ASPHYXIA ON PERIPHERAL OXYGENATION AND PERFUSION IN NEONATES}

doi:10.1136/archdischild-2012-302724.0026

G Pichler, N Tax, C Binder, M Pocivalnik, E Pichler-Stachl, W Müller, B Urlesberger. Medical University of Graz, Graz, Austria

Objective The aim was to investigate the influence of perinatal asphyxia on peripheral oxygenation and perfusion in neonates in a prospective observational study.

Methods Neonates with over 34 weeks gestational age and birth weight $>2000 \mathrm{~g}$ without sepsis or connatal malformations were included. Neonates with an umbilical artery $\mathrm{pH}$ of $(\mathrm{UapH}) \leq 7.15$ and 5 minute APGAR score $\leq 6$ were investigated. Neonates with an $\mathrm{UapH} \geq 7.15$, and 5 minute APGAR score $\geq 7$ served as control group.

Peripheral muscle near infrared spectroscopy (NIRS) measurement in combination with venous occlusion was performed once in the first 48 hours after birth. Tissue oxygenation index (TOI), mixed venous oxygen saturation (SvO2), fractional oxygen extraction (FOE), haemoglobin flow (Hbflow), oxygen delivery (DO2) and oxygen consumption (VO2) were assessed. Furthermore arterial oxygen saturation, heart rate, blood pressure und temperatures were measured. UapH was correlated to NIRS parameters.

Results Eight asphyxiated neonates were included. In the asphyxiated group significant correlations between $\mathrm{UapH}$ and $\mathrm{DO} 2$ $(\mathrm{r}=0.78)$, VO2 $(\mathrm{r}=0.80)$ and FOE $(\mathrm{r}=-0.75)$ were found. The asphyxiated neonates were compared to 30 neonates in the control group. TOI $(67.7 \pm 5.5 \%)$ and DO2 $(29.0 \pm 14.2 \mu \mathrm{mol} / 100 / 1 / \mathrm{min})$ were significantly lower in asphyxiated neonates compared to the controls (TOI 71.8 $\pm 4.9 \%$, DO2 43.9 $\pm 16.9 \mu \mathrm{mol} / 100 / \mathrm{l} / \mathrm{min}$ ), FOE was significantly higher $(0.33 \pm 0.05)$ compared to the controls $(0.28 \pm 0.06)$. No correlation of UapH with NIRS parameters was observed in the control group.

Conclusion Peripheral oxygenation and perfusion measured with NIRS is compromised in neonates with perinatal asphyxia with worsening of parameters with severity of asphyxia.

\section{METABOLIC OUTCOMES OF CHILDREN AND ADULTS BORN PRETERM}

doi:10.1136/archdischild-2012-302724.0027

${ }^{1} \mathrm{~N}$ Modi, ${ }^{2} \mathrm{~A}$ Singhal, ${ }^{2} \mathrm{M}$ Fewtrell, ${ }^{1} \mathrm{M}$ Hyde. ${ }^{1}$ Imperial College London; ${ }^{2}$ Institute of Child Health, London, UK

Babies below 37 weeks gestation now account for $9-12 \%$ of all births, and babies $<32$ weeks gestation for $1-2 \%$. Survival is also rising, and the expectation of life-long health. Preterm babies appear to be at substantially greater risk of features of the metabolic syndrome. For example it is estimated that currently 1 in 15 newly diagnosed hypertensives will have been born preterm. Early nutrition is the likely candiate mediator of long-term effects as well as a potential attenuator of aberrant trajectories of metabolic health. We will summarise research addressing childhood and adult metabolic health following preterm birth, the evidence that early nutrition and preterm growth affects risk for the metabolic syndrome. We will discuss methodological aspects of establishing causal relationships between infant feeding and later health outcomes, the pros and cons of observational versus randomized trials, practical issues in conducting infant nutrition studies/trials and performing longterm follow-up studies. We will provide an overview of the wide range of non-invasive technologies available for the identification of biomarkers in infants (including metabolomic technologies (using multi-component NMR/GCMS/LCMS platforms), genetic analyses using buccal swabs, and in vivo magnetic resonance imaging and spectroscopy to measure metabolites in the liver and brain.

\section{THE QUEST TO IDENTIFY BIOMARKERS OF LONG-TERM OUTCOME IN THE NEWBORN}

doi:10.1136/archdischild-2012-302724.0028

MJ Hyde, N Modi. Neonatal Medicine, Imperial College London, London, UK

We know that many events in the perinatal period have lifelong health implications. Work from animal models, and limited human data, suggest that deleterious long-term outcomes could be prevented by intervention in the neonatal period. While many studies of early life interventions are currently ongoing, outcome measures have to be in the short to medium term, even though the greatest impact of these interventions may not become apparent until well into adulthood. We are currently looking for biomarkers which can be measured non-invasively in the short term, but which have a strong association with long term outcome and may therefore provide indications of what the long-term effect of our experimental intervention may be. Methods currently being investigated include metabolomic technologies (using multi-component NMR/GCMS/ LCMS platforms), genetic analysis using buccal swabs; in vivo magnetic resonance imaging and spectroscopy to measure metabolites in the liver and brain.

\section{HEAD INJURIES}

doi:10.1136/archdischild-2012-302724.0029

L Tume. PICU, Alder Hey Childrens' Hospital, Liverpool, UK

This lecture will provide an overview of the pathophysiology of severe traumatic brain injury in children and the intensive care management of a child with severe TBI, focusing on the new WFPICS guidance (2012) and tiers of evidence. It will then present evidence for the nursing management of these children.

\section{BIRTH AND REBIRTH - PARENTAL EXPERIENCES OF THEIR NEWBORN INFANTS TREATED WITH HYPOTHERMIA FOLLOWING PERINATAL ASPHYXIA}

doi:10.1136/archdischild-2012-302724.0030

'S Kokkonen Nassef, ${ }^{2} \mathrm{M}$ Blennow, ${ }^{3} \mathrm{M}$ Jirwe. ${ }^{1} \mathrm{NICU}$, Karolinska University Hospital Huddinge; '2Department of Clinical Sciences, Intervention and Technology, Karolinska Institutet, Stockholm, Sweden; ${ }^{3}$ Department of Nursing, Division of Neurobiology, Care Sciences and Society, Karolinska Institutet, Stockholm, Sweden

The normal caring and nursing of newborn infants is to keep them warm and close to their parents. Fullterm newborn infants suffering from perinatal asphyxia are treated with induced hypothermia treatment (IHT) for three days at the Neonatal Intensive Care Unit (NICU) in order to prevent or decrease brain damages. The design of the study was a descriptive qualitative study. The aim was to describe and understand experiences of parents whose newborn infants were treated with IHT following perinatal asphyxia. A total of ten parents participated in the study, seven mothers and three fathers. Open-ended recorded interviews were conducted 4-12 months after the birth of their infants. Inductive content analysis 